\section{ATENÇÃO INTEGRAL À SAÚDE DO HOMEM: UM DESAFIO NA ATENÇÃO BÁSICA}

\author{
Comprehensiveness in men's health care: a challenge in \\ primary care
}

\author{
Atención integral a la salud del hombre: un desafío de la \\ atención básica
}

\section{RESUMO}

Objetivo: Compreender a percepção dos profissionais de saúde sobre a Política Nacional de Atenção Integral à Saúde do Homem (PNAISH). Métodos: Trata-se de um estudo qualitativo e descritivo, realizado com 11 profissionais da Unidade Básica de Saúde, entre fevereiro e abril de 2015, no Município de Quixadá/CE. A amostra foi escolhida aleatoriamente até que houvesse a saturação teórica. A partir da temática, indagou-se algumas questões norteadoras sobre definição, objetivos, princípios, diretrizes, ações, dificuldade e potencialidade da implementação da PNAISH. A análise dos dados foi baseada na técnica do Discurso do Sujeito Coletivo. Resultados: Ficou evidenciado que os profissionais entrevistados não conhecem a PNAISH e poucos compreendem a sua importância para o acompanhamento integral do homem. Há várias dificuldades para a implementação da PNAISH, tais como a deficiência de recursos materiais e humanos, e ainda a falta de interesse do próprio homem em cuidar da sua saúde. Conclusão: Ficou evidenciado que grande parte dos profissionais entrevistados não conhecem a PNAISH e poucos compreendem a sua importância para o acompanhamento integral do homem. O cuidado do homem é um processo dinâmico e complexo, tendo a PNAISH fundamental importância para o acolhimento da população masculina nos serviços de saúde, porém urge ressaltar que a referida política deve ter uma maior divulgação voltada aos profissionais da saúde.

Descritores: Centros de Saúde; Políticas Públicas de Saúde; Atenção Primária à Saúde; Saúde do Homem.

\section{ABSTRACT}

Objective: To understand the health professionals' perception of the National Policy for Integral Attention to Men's Health (PNAISH - Politica Nacional de Atenção Integral à Saúde do Homem). Methods: This is a qualitative and descriptive study, performed with 11 professionals of the Primary Health Care Unit, between February and April 2015 in the city of Quixadá, CE. The sample was randomly selected until the theoretical saturation was reached. From the theme, some guiding questions were asked, addressing the definition, objectives, principles, guidelines, actions, difficulties and potential of the implementation of the PNAISH. Data analysis was based on the Collective Subject Discourse technique. Results: It was evidenced that the health professionals interviewed do not know the PNAISH and few of them realize its importance for the comprehensive attention to men. There are several difficulties in the implementation of PNAISH, such as the deficiency in material and human resources, in addition to the lack of interest on the part of the man himself in caring for his health. Conclusion: Man's care is a complex and dynamic process, in which the PNAISH has a fundamental significance for the embracement of the male population in the health services. However, it is noteworthy that this policy should be more publicized among the health professionals. The study revealed that most of the professionals interviewed do not know the PNAISH and few understand its importance to the comprehensive integrated evaluation of man.

Descriptors: Health Centers; Public Health Policy; Primary Health Care; Men's Health.
Artigo Original

\author{
Liana Maria Rocha Carneiro(1) \\ Mayara Paz Albino dos Santos ${ }^{(2)}$ \\ Raimunda Hermelinda Maia \\ Macena $^{(2)}$ \\ Thiago Brasileiro de \\ Vasconcelos $^{(2)}$
}

1) Escola de Saúde Pública do Ceará - ESP CE - Fortaleza (CE) - Brasil

2) Universidade Federal do Ceará - UFC Fortaleza (CE) - Brasil
Recebido em: 15/06/2016 Revisado em: 14/08/2016 Aceito em: 20/10/2016 


\section{RESUMEN}

Objetivo: Comprender la percepción de los profesionales sanitarios sobre la Política Nacional de Atención Integral a la Salud del Hombre (PNAISH). Métodos: Se trata de un estudio cualitativo y descriptivo realizado con 11 profesionales de la Unidad Básica de Salud entre febrero y abril de 2015 en el Municipio de Quixadá/CE. Se escogió la muestra de modo aleatorio hasta que hubiera la saturación de la teoría. A partir de la temática, algunas cuestiones norteadoras respecto la definición, los objetivos, los principios, las directrices, las acciones, la dificultad y potencialidad para la implementación de la PNAISH fueron realizadas. El análisis de los datos estuvo basado en la técnica del Discurso del Sujeto Colectivo. Resultados: Se evidenció que los profesionales entrevistados no conocen la PNAISH y pocos de ellos comprenden su importancia para el seguimiento integral del hombre. Hay varias dificultades para la implementación de la PNAISH tales como la carencia de recursos materiales y humanos y también la falta de interés de los hombres para el cuidado de su salud. Conclusión: Se evidenció que la mayoría de los profesionales entrevistados no conocen la PNAISH y pocos comprenden su importancia para el seguimiento integral del hombre. El cuidado del hombre es un proceso dinámico y complejo con fundamental importancia de la PNAISH para la acogida de la población masculina en los servicios de salud pero hace falta resaltar que la referida política debe ser más difundida entre los profesionales sanitarios.

Descriptores: Centros de Salud; Politicas Públicas de Salud; Atención Primaria de Salud; Salud del Hombre.

\section{INTRODUÇÃO}

Sabe-se que a população masculina frequenta menos os serviços de Atenção Primária à Saúde do que a população feminina. Tal comportamento é influenciado por diversos fatores que levam a um aumento na morbimortalidade do homem, colaborando para a má qualidade de saúde desse público $^{(1,2)}$.

Tais fatores são de natureza social, comportamental, cultural, pessoal, dentre outros, em que colaboram as poucas ações de promoção da saúde e prevenção de doenças direcionadas ao público masculino, fato que pode ser influenciado pelo despreparo dos profissionais de saúde, inércia das políticas públicas de saúde no Brasil e pouco apoio estrutural a fim de garantir a continuidade das ações $^{(1,3-6)}$.

Desde 2009, foi lançada, no Brasil, a Política Nacional de Atenção Integral à Saúde do Homem (PNAISH), específica para o público masculino, representando uma inovação no campo da saúde ${ }^{(7)}$. De acordo com a PNAISH, estudos demostraram que $60 \%$ das mortes no país correspondiam a homens e que a expectativa de vida dessa população seria
7,6 anos menor que as mulheres. Dessa forma, a política busca chamar a atenção dos homens, para que cuidem da sua saúde, e da população em geral, para que compreendam a realidade singular masculina ${ }^{(8)}$.

Sendo assim, a PNAISH apresenta como área de atenção a população masculina de 20 a 59 anos, tem como objetivo melhorar as condições de saúde dos homens e reduzir a morbimortalidade dessa população através de ações de promoção, prevenção e proteção básica à saúde, facilitando o acesso aos serviços de saúde, qualificando e humanizando a atenção integral à saúde do homem ${ }^{(8)}$. Diante disso, é importante destacar que a PNAISH veio despertar e sensibilizar, tanto os homens quanto os profissionais da saúde, sobre a importância do cuidado da saúde do homem, buscando uma melhor compreensão do processo saúdedoença masculino e o fortalecimento da Atenção Primária ${ }^{(9)}$.

Durante a experiência na Residência Integrada com Ênfase em Saúde da Família e Comunidade, teve-se a oportunidade de uma maior apropriação da PNAISH e percebeu-se, tanto no processo de territorialização quanto na assistência de enfermagem, a ausência do público masculino e o número reduzido de ações desenvolvidas sobre saúde do homem na unidade. Assim, objetivouse compreender a percepção dos profissionais de saúde sobre a Política Nacional de Atenção Integral à Saúde do Homem, em razão desses profissionais atuarem na Atenção Básica, considerada a principal "porta de entrada" do Sistema Único de Saúde (SUS), e serem fundamentais para o desenvolvimento de ações que busquem a promoção, prevenção e recuperação da saúde do homem.

\section{MÉTODOS}

Estudo com abordagem qualitativa do tipo descritivo, realizado entre fevereiro e abril de 2015, em uma unidade básica de saúde (UBS) do município de Quixadá, Ceará. $\mathrm{O}$ uso dessa metodologia justifica-se por se buscar compreender e contextualizar a percepção dos profissionais de saúde sobre a Política Nacional de Atenção Integral a Saúde do Homem, já que a pesquisa qualitativa procura compreender o processo pelo qual as pessoas constroem significados e escrevem o que são, privilegiando os aspectos subjetivos do sujeito ${ }^{(10)}$.

A UBS pesquisada apresenta um território geograficamente extenso e diverso, sendo possível detectar divergências culturais, econômicas, geográficas, políticas e epidemiológicas, e atende a uma população estimada de 3500 famílias.

A população do estudo foi composta pelos profissionais da saúde da UBS, contabilizados em janeiro de 2015 em 32 profissionais, dentre os quais haviam enfermeiros, médico, dentistas, auxiliar de saúde bucal, técnicos de enfermagem 
e agentes comunitários de saúde, os quais desenvolvem suas atividades junto à equipe da Estratégia de Saúde da Família (ESF). A amostra foi escolhida aleatoriamente até que houvesse uma saturação teórica, ou seja, quando as informações fornecidas pelos participantes da pesquisa passassem a apresentar, na avaliação dos pesquisadores, uma certa redundância, não mais contribuindo na reflexão teórica ${ }^{(11)}$. Ressalta-se que todas as entrevistas foram realizadas por um único pesquisador com experiência nesse tipo de abordagem.

Os sujeitos foram considerados em número suficiente para gerar a reincidência das informações, com inclusões sucessivas e satisfatórias de profissionais para uma discussão densa das questões formuladas, ou seja, a quantidade numérica de entrevistados utilizada no estudo não interferiu na qualidade dos resultados obtidos, pois a pesquisa qualitativa enfoca as informações e o aprofundamento na análise dos $\operatorname{dados}^{(12)}$. Assim, a amostra não buscou uma representatividade numérica, mas sim um aprofundamento da temática, sendo composta por 11 profissionais da saúde da UBS pesquisada.

Foram utilizados como critérios de inclusão: trabalhar na UBS pesquisada, possuir condições de responder por si próprio as perguntas que foram realizadas durante o estudo, ser maior de 18 anos e assinar previamente o Termo de Consentimento Livre e Esclarecido. Foram excluídos os sujeitos que não responderam uma ou mais questões e que não estavam em condições físicas e/ou psicológicas para participar do estudo.

Como instrumentos e procedimentos, foram empregados a observação não participante e o roteiro de entrevista semiestruturado, juntamente com o uso de gravador (Sony ${ }^{\circledR}$, modelo ICD PX 240) e diário de campo.

A análise dos dados foi baseada na técnica Discurso do Sujeito Coletivo (DSC), que considera a discursividade como característica principal do pensamento coletivo, preservando-a em todas as etapas da pesquisa. Essa técnica metodológica busca organizar os dados qualitativos de naturezas diferentes, apresentando como proposta a análise do material coletado, destacando as ideias centrais (IC) e as suas expressões-chaves, compondo discursos na primeira pessoa do singular ${ }^{(13,14)}$.
As informações coletadas e transcritas foram organizadas em um quadro-síntese destacando as ideias centrais referentes aos seguintes questionamentos: Qual a sua opinião sobre a saúde do homem? Você conhece a PNAISH? Caso você conheça, o que você sabe sobre a PNAISH (objetivos, princípios e diretrizes)? Quais ações de saúde são realizadas para essa população na UBS? Quais as dificuldades enfrentadas para a implementação da Política Nacional de Atenção Integral a Saúde do Homem na UBS? Quais as potencialidades para a implementação da Política Nacional de Atenção Integral à Saúde do Homem na UBS? O que poderia ser realizado para a população masculina na UBS?

O estudo foi aprovado pelo Comitê de Ética em Pesquisa da Escola de Saúde Pública do Ceará (ESP/CE), sob o parecer $n^{\circ} .962 .460$.

\section{RESULTADOS E DISCUSSÃO}

Para a apresentação dos resultados dessa pesquisa, os questionamentos foram divididos em 6 grupos que resultaram em DSC enumerados de 1 a 6 com especificações das IC, que também foram enumeradas de acordo com o número de variáveis que emergiram em cada discurso.

A amostra foi composta por 11 profissionais da saúde da UBS pesquisada e os resultados a seguir destacam as ideias centrais dos discursos do sujeito coletivo.

Em relação à IC 1 (Quadro I), os profissionais de saúde consideram que o homem não cuida da própria saúde, pois eles acreditam que são mais resistentes às doenças e buscam os serviços de saúde apenas quando estão com dor, conforme ilustrado no DSC a seguir: "O homem não gosta de cuidar da saúde. O homem em si, ele procura muito pouco o posto de saúde, só procura mais quando está com dor. Pensa que é mais resistente do que a mulher, mas está mais vulnerável ao adoecimento, aos agravos, aos acometimentos de saúde" (DSC 1, IC 1).

Essa ideia destacada pelos profissionais é característica da maioria dos homens que adotam um comportamento baseado em um modelo de masculinidade que o expõe a atitudes pouco saudáveis, que podem resultar em agravos à saúde, contudo, alguns autores ${ }^{(15)}$ destacam

Quadro I - Quadro-síntese sobre as Ideias Centrais referentes ao questionamento: Qual a sua opinião sobre a saúde do homem? Quixadá, Ceará, Brasil, 2015.

\section{DSC1}

\begin{tabular}{ccccc}
$\begin{array}{c}\text { Ideia central 1: O } \\
\text { Homem não cuida da } \\
\text { sua saúde }\end{array}$ & $\begin{array}{c}\text { Ideia central 2: } \\
\text { É importante }\end{array}$ & $\begin{array}{c}\text { Ideia central } \\
\text { 3: Igualdade no } \\
\text { acompanhamento }\end{array}$ & $\begin{array}{c}\text { Ideia central 4: } \\
\text { Dificuldade de trabalhar } \\
\text { saúde do homem }\end{array}$ & $\begin{array}{c}\text { Ideia central 5: } \\
\text { Saúde do homem em } \\
\text { família }\end{array}$ \\
\hline
\end{tabular}


que é imprescindível evitar reduzir as análises apenas as características hegemônicas, é necessário ver o homem com o foco nas suas relações sociais e interações. O DSC 1 referente à IC 3 (Quadro I) corrobora com estes autores quando afirmam que: "Quando se trata de saúde não existe homem nem mulher, existe um ser humano que necessita de todos os cuidados".

Outros autores ${ }^{(1,16)}$ afirmam que a masculinidade construída socioculturalmente ainda prevalece, trazendo a ideia de que o homem nunca adoece, de invulnerabilidade, de comportamento de risco e de sexualidade instintiva e incontrolável, associada a uma desvalorização do autocuidado. Isso resulta em dificuldades de verbalização das próprias necessidades de saúde, já que essa atitude pode demonstrar fraqueza e feminilização. Dessa maneira, o homem não se cuida e só procura o serviço de saúde nas últimas consequências.

No DSC 1, IC 2 (Quadro I), os profissionais consideram importante a saúde do homem, porém há pouco incentivo para se trabalhar com esse público-alvo, que necessita de atenção e cuidado por parte da equipe de saúde: "Acho importante ter esse programa para o homem, mas não vejo incentivo. O homem é um público-alvo e nós, profissionais da saúde, devemos estar atentos a esse público-alvo".

Durante muito tempo, os serviços e as estratégias de saúde e comunicação privilegiaram as políticas e ações voltadas para a promoção da saúde da criança, do adolescente, da mulher e do idoso, sendo o homem excluído dessas políticas públicas, ficando à margem desses cuidados em saúde ${ }^{(3)}$. Várias são as suposições para tal fato, como a pouca presença dos homens nas UBS, a identidade masculina associada à desvalorização do autocuidado e à preocupação insuficiente com a saúde, e a procura de serviços de pronto-atendimento ${ }^{(17)}$.

Somente em 2009, o Estado reconheceu a condição de saúde dos homens no Brasil como um problema de saúde pública, sendo o homem destacado como público-alvo das ações em saúde com o lançamento e a implementação da Política Nacional de Atenção Integral à Saúde do Homem. A PNAISH objetiva promover a melhoria das condições de saúde da população masculina do Brasil, contribuindo, de modo efetivo, para a redução da morbidade e mortalidade dessa população mediante o enfrentamento racional dos fatores de risco e por via da facilitação do acesso às ações e aos serviços de assistência integral à saúde.

Essa política destaca a necessidade de mudanças nos padrões que correspondem à percepção da população masculina referente ao cuidado com a sua saúde e a saúde de sua família, e apresenta como desafio a mobilização da população masculina pela luta de seus direitos à saúde ${ }^{(8)}$.

Outro aspecto a destacar no DSC 1 referente à IC 4 (Quadro I): "Na Estratégia Saúde da Família, eu vejo que não temos conseguido atingir essa demanda de realizar atividades mais voltadas para a saúde do homem", é evidente a dificuldade de trabalhar a saúde masculina na ESF, principalmente pelo fato do homem buscar primeiramente os serviços de média e alta complexidade. Segundo o Ministério da Saúde ${ }^{(8)}$, os homens adentram o sistema de saúde através da atenção especializada, indicando que, quando procuram os serviços de saúde, encontramse com a saúde já comprometida e a doença instalada, o que torna necessário o fortalecimento e a qualificação da atenção primária, que é a porta de entrada do Sistema Único de Saúde, uma vez que garante a promoção da saúde e a prevenção dos problemas evitáveis.

Nesse contexto, os profissionais também destacaram no DSC 1, IC 5 (Quadro I), a influência da saúde do homem em relação a sua função no contexto familiar, como sendo o responsável por proteger, zelar e conduzir a família: " $A$ saúde do homem influencia muito no contexto de uma família". Corroborando esse achado, um estudo enfatiza a necessidade de uma maior participação dos homens no cuidado da saúde ${ }^{(17)}$.

No DSC 2, IC 1 (Quadro II), os profissionais referem não conhecer a PNAISH, não sabendo da sua existência. Isso pode estar relacionado com o fato de ser uma política nova, que foi lançada somente em 2009, e com a falta de capacitação dos profissionais da saúde que trabalham na atenção básica. Um estudo ${ }^{(18)}$ realizado com profissionais da ESF destacou a carência em relação ao cuidado da saúde do homem, pois, em geral, há uma reduzida oferta de assistência e de atividades de promoção e prevenção para essa população masculina.

Quadro II - Quadro-síntese sobre as Ideias Centrais referentes ao questionamento: Você conhece a PNAISH? Caso você conheça, o que você sabe sobre a PNAISH (objetivos, princípios e diretrizes)? Quixadá, Ceará, Brasil, 2015.

DSC 2

Ideia central 1:

Não conheço
Ideia central 2:

Conheço, mas nunca li
Ideia central 3: Conheço, já li, mas é necessário maior apropriação
Ideia central 4:

Princípios e diretrizes 
Resposta semelhante pode ser destacada no DSC 2, IC 2 e 3 (Quadro II), em que os profissionais relatam saber sobre a política, mas com limitações, não conhecendo os objetivos, princípios e diretrizes que norteiam a política de saúde do homem. Assim, ficou evidenciado que há insuficiência de dados e conhecimento sobre a PNAISH por parte dos profissionais de saúde entrevistados.

É importante destacar que há uma fragilidade nas informações fornecidas aos profissionais da saúde em relação à PNAISH, visto que muitas vezes nem são repassadas pela Secretaria de Saúde. A deficiência desses conhecimentos também ocorre pela falta de sensibilização dos profissionais em buscar informações para o aperfeiçoamento e desenvolvimento de ações direcionadas ao homem, pois, para implementar a PNAISH, os profissionais devem conhecer seus objetivos, princípios e diretrizes, tendo como premissa o planejamento de ações de promoção em saúde voltadas a essa populaçãa ${ }^{(19)}$.

Outro aspecto a destacar, no DSC 2, é a IC 4 (Quadro II), em que apenas um entrevistado afirma conhecer os princípios e diretrizes da PNAISH, mas refere a necessidade de uma maior apropriação dessa política, que, apesar de ter sido lançada recentemente, é fundamental para que inicie mudanças no acolhimento à população masculina nos serviços de saúde ${ }^{(4)}$. Alguns autores ${ }^{(5)}$ enfatizam a importância da qualificação dos profissionais que trabalham na atenção básica, principalmente pelos avanços teóricos, organizacionais e políticos que ocorreram nos últimos anos, assim como a diversidade de atuação dos profissionais.

Quadro III - Quadro-síntese com as Ideias Centrais da indagação: Quais ações de saúde são realizadas para essa população na UBS? Quixadá, Ceará, Brasil, 2015.

\begin{tabular}{|c|c|c|c|}
\hline \multicolumn{4}{|c|}{ DSC 3} \\
\hline $\begin{array}{c}\text { Ideia central 1: Campanha } \\
\text { do Novembro azul }\end{array}$ & $\begin{array}{l}\text { Ideia central 2: } \\
\text { Difícil trabalhar o } \\
\text { homem }\end{array}$ & $\begin{array}{c}\text { Ideia central 3: } \\
\text { O programa de hipertensão e } \\
\text { diabetes }\end{array}$ & $\begin{array}{c}\text { Ideia central 4: } \\
\text { Especificamente não há } \\
\text { ações }\end{array}$ \\
\hline
\end{tabular}

As IC 1 e 3 (Quadro III) destacam algumas ações de saúde direcionadas ao homem, realizadas na UBS, como a campanha Novembro Azul, de caráter educativo, específica ao público masculino, que trabalha a prevenção do câncer de próstata bem como o atendimento médico e de enfermagem ao paciente com hipertensão e diabetes. Porém, esta última ação não é específica ao público masculino: "Campanha do mês de novembro com ações voltadas ao CA de próstata, incentivando a fazer o exame de PSA e de toque" (DSC 3, IC 1) e "Acompanhamento médico e de enfermagem no controle da hipertensão e diabetes, é quando a gente tem um maior número de homens" (DSC 3, IC 3).

$\mathrm{Na}$ atenção básica, pode-se perceber uma visão restrita acerca da saúde do homem, com uma tendência de restringir, principalmente, aos problemas da próstata, contrapondo-se aos princípios e diretrizes da PNAISH, que orienta um cuidado integral da saúde do homem ${ }^{(4,17)}$. Em um estudo de revisão ${ }^{(9)}$, na qual abordou a temática da saúde do homem e masculinidades na PNAISH através de estudos publicados entre os anos 2005 e 2011, os autores destacaram a importância do exame de toque retal para prevenção do câncer de próstata, não obstante, sem diminuir a relevância da prevenção de acidentes de trânsito e da violência que causam sofrimento para os homens e para as famílias.

A IC 2 (Quadro III) considera, com base no discurso dos profissionais, que é muito difícil trabalhar saúde do homem.
A inclusão dos homens nas ações de saúde é um desafio para o sistema de saúde, pois a saúde do homem ainda não é trabalhada de maneira integral ${ }^{(20)}$. O cuidado e a atenção à saúde da população masculina é um processo dinâmico e complexo $^{(3)}$. Deste modo, os profissionais da saúde devem ser capacitados para trabalhar com esse público, tendo o cuidado com suas particularidades e preocupação com a integralidade no cuidado ${ }^{(18)}$.

Um estudo recente( ${ }^{(21)}$ destaca a importância da potencialização da participação do homem na realização dos exames clínicos de rotina, na estratégia do pré-natal na paternidade e no planejamento familiar, contudo, orientam a necessidade de atender suas demandas, pois são impacientes, gostam de estímulos materiais em troca da participação nas atividades (por exemplo, preservativos, material de higiene, dentre outros) e manutenção do vínculo com os profissionais, estratégias que remetem zelo, atenção e satisfação com os serviços prestados.

Já em relação à IC 4 (Quadro III), os profissionais referem que não há ações específicas para o homem na UBS. $\mathrm{O}$ atendimento em geral inclui também o atendimento ao homem, porém não são desenvolvidas atividades características que promovam a saúde integral do público masculino. Considerável parte do atendimento da atenção básica prioriza grupos populacionais considerados mais vulneráveis, por meio de ações desenvolvidas e direcionadas 
à saúde da mulher, da criança e do idoso, pouco favorecendo a atenção à saúde do homem ${ }^{(1,4,18)}$. Dessa maneira, uma atenção específica a esse público proporcionaria aos profissionais e ao próprio homem um melhor conhecimento das especificidades e reais necessidades masculinas ${ }^{(22)}$.

Sendo assim, percebeu-se que não são realizadas ações que contemplem especificamente a coletividade masculina, não considerando as reais necessidades desse públicoalvo e tampouco os indicadores de saúde referenciados na PNAISH, sendo pontuais as atividades direcionadas especificamente ao homem. Dados que vão ao encontro de outro estudo $^{(1)}$, realizado na cidade de Quixadá/CE, com a opinião dos homens sobre a atenção primária, em que eles destacaram que o homem procura os serviços de saúde com fim curativista, para resolução de doenças já instaladas, e apresentam necessidades referentes à orientação sobre PNAISH, diálogo com os profissionais de saúde, atendimento especializado e alternativas que facilitem o seu acesso ao serviço de saúde.

Um estudo de $2013^{(23)}$ acrescenta a importância de romper com a concepção de homem, hoje reduzida a “corpos com pênis e próstata". É preciso mudar o foco simplista de somente julgá-los como culpados pelas suas escolhas, tratar a saúde do homem como algo complexo e multifatorial. Para tanto, a prevenção e promoção da saúde destacam-se como medidas importantes e concretas no desafio da integralidade e assistência humanizada.

Quadro IV - Quadro-síntese sobre as Ideias Centrais referentes ao questionamento: Quais as dificuldades enfrentadas para a implementação da Política Nacional de Atenção Integral a Saúde do Homem na UBS? Quixadá, Ceará, Brasil, 2015.

\begin{tabular}{|ccccc|}
\hline \multicolumn{5}{c|}{ DSC 4 } \\
\hline Ideia central 1: & Ideia central 2: & Ideia central 3: & Ideia central 4: Falta & Ideia central 5: Falta \\
Falta de recursos & Falta de interesse do & Não há dificuldades & conscientização e & de conhecimento da \\
materiais e humanos & homem & & PNAISH \\
\hline
\end{tabular}

Na IC 1 (Quadro IV), os profissionais destacam a falta de recursos materiais e humanos como dificuldades para implementar a PNAISH na UBS. Os recursos não chegam a UBS e, se existem, não são repassados. A falta de espaço na unidade para a realização de ações educativas, como palestras e oficinas, também é um problema. Dessa forma, é necessário haver uma melhora por parte do poder público, como o repasse financeiro com planejamento e a contratação de mais profissionais.

A falta de estrutura e sistematização dos serviços de saúde relacionados aos recursos humanos e materiais, bem como o espaço físico adequado para o acolhimento de qualidade da população masculina, são fatores que reforçam a baixa procura dos homens pelos serviços de atenção primária ${ }^{(4)}$.

Em relação à IC 2 (Quadro IV), verifica-se que a falta de interesse do próprio homem também é um problema para implementar a PNAISH. Os profissionais afirmam que há uma dificuldade em chegar até o homem para conscientizálo de ir à UBS e cuidar da sua saúde, ou seja, os profissionais acreditam que uma grande dificuldade para o trabalho com a saúde do homem é fazer com que este chegue ao serviço antes de adoecerem. Essa característica do homem está relacionada, muitas vezes, às questões culturais e de gênero, fazendo com que ele busque os serviços de saúde apenas quando está doente ${ }^{(1)}$.
Os homens apresentam uma maior resistência para buscar os serviços de saúde, principalmente a atenção básica, por acreditarem que a prevenção e o autocuidado estão relacionadas à fragilidade, ao contrário da exposição a situações de risco e invulnerabilidade a que está relacionada uma visão hegemônica de masculinidade ${ }^{(24)}$. Porém, é importante ressaltar também que não só a questão cultural dificulta a adesão do homem aos serviços de saúde, mas as questões de acesso ao serviço também constituem uma barreira $^{(9)}$.

Um discurso diferenciado emerge na IC 3 (Quadro IV), que destaca que não há dificuldades para implementar a PNAISH na UBS. Nesse discurso, os profissionais afirmam que, se houver o empenho de toda a equipe, não vai haver dificuldades para realizar ações de saúde do homem e, assim, implementar a política em questão. $\mathrm{O}$ trabalho em equipe é importante e apresenta vantagem em relação ao trabalho individual, pois conhecimentos, decisões e responsabilidades são compartilhados e, dessa forma, possibilitam a realização de um trabalho integrado e produtivo ${ }^{(25)}$.

Já em relação à IC 3 e 4 (Quadro IV), o discurso apresenta como dificuldades a falta de sensibilização, conscientização e conhecimento dos profissionais que atuam na saúde. Pode-se perceber, no estudo, que poucos profissionais de saúde estão preparados para trabalhar com 
o público masculino e isso está relacionado principalmente à falta de capacitações contínuas sobre a saúde do homem, dificultando o acolhimento e o atendimento integral dessa população. Como a maioria dos profissionais não estão capacitados, há poucas ações na atenção básica específicas do gênero masculino ${ }^{(4,18)}$.

Segundo o Ministério da Saúde ${ }^{(8)}$, a capacitação técnica dos profissionais de saúde para o atendimento do homem e a disponibilidade de insumos, equipamentos e materiais educativos são princípios que norteiam a PNAISH. Dessa maneira, faz-se necessária, como preconiza uma das diretrizes da política do homem, a reorganização das ações de saúde através de uma proposta inclusiva, na qual os homens considerem os serviços de saúde também como espaços masculinos e, por sua vez, os serviços de saúde reconheçam os homens como sujeitos que necessitam de cuidados.

Quadro V - Quadro-síntese sobre as Ideias Centrais referentes ao questionamento: Quais as potencialidades para a implementação da Política Nacional de Atenção Integral à Saúde do Homem na UBS? Quixadá, Ceará, Brasil, 2015.

\begin{tabular}{|ccc|}
\hline & DSC 5 \\
\hline Ideia central 1: & Ideia central 2: & Ideia central 3: \\
Equipe comprometida & Residência Multiprofissional & Acesso \\
\hline
\end{tabular}

Na IC 1 (Quadro V), os profissionais destacam como potencialidade para a implementação da PNAISH o comprometimento e o empenho da equipe da UBS, que driblam todas as dificuldades e fazem o possível para a promoção de uma saúde com qualidade para a comunidade:

"Primeiro, assim, a força de vontade dos profissionais, que eu acho assim, que as meninas, elas driblam todas as dificuldades e fazem o possível, não só para o homem, mas para todos os programas que tem aqui no posto. Cuidado e a preocupação que a equipe tem em sempre procurar dar o melhor para a comunidade. Temos uma equipe muito boa, empenhada e compromissada com o usuário" (DSC 1, IC $1)$.

A IC 2 (Quadro V) considera, com base nos discursos dos profissionais, a equipe de residência multiprofissional como uma potencialidade para a implementação da saúde do homem na UBS através da PNAISH. A residência multiprofissional é uma potente estratégia de educação permanente que trabalha junto à equipe de saúde da família, promovendo a interprofissionalidade, a integralidade e a intersetorialidade na atenção, gestão e participação popular. Dessa maneira, irá ajudar a promover a saúde do homem na unidade de saúde onde estão atuando.
Já em relação à IC 3 (Quadro V), o acesso é indicado como uma potencialidade para a implementação da PNAISH: "a boa localização da unidade e o fácil acesso permitem que o homem, como também a sua família, busque serviços de saúde". O acesso é importante para a implementação de práticas em serviços de saúde na perspectiva da integralidade do cuidado, pois possibilita a realização de um cuidado com base nas necessidades, tem relação com a resolubilidade e ultrapassa a dimensão geográfica, compreendendo aspectos de ordem econômica, cultural e funcional de oferta de serviços ${ }^{(22,26)}$.

Na IC 1 (Quadro VI), os profissionais consideram como ações propostas para a população masculina aproveitar a oportunidade quando o homem vai à unidade de saúde para acolhê-los e sensibilizá-los sobre a importância do autocuidado e da prevenção de doenças.

A inclusão e o acolhimento dos homens nos serviços de saúde são importantes para que eles se sintam participantes do cuidado, e ações devem ser desenvolvidas pela atenção básica, sendo essa um local privilegiado para o desenvolvimento dessas práticas devido à sua maior proximidade com a comunidade e por voltar-se para ações preventivas e de promoção à saúde ${ }^{(18,27)}$.

Quadro VI - Quadro-síntese sobre as Ideias Centrais referentes ao questionamento: O que poderia ser realizado para a população masculina na UBS? Quixadá, Ceará, Brasil, 2015.

\section{DSC 6}

Ideia central 1:

Aproveitar a oportunidade (acolhimento)
Ideia central 2:

Promoção da Saúde
Ideia central 3: Ir

onde o homem está
Ideia central 4:

Dia do homem
Ideia central 5:

Capacitação dos

profissionais 
Na IC 2 (Quadro VI), os profissionais destacaram que deveriam ser realizadas e intensificadas as ações de promoção da saúde e de prevenção de doenças direcionadas ao público masculino através de campanhas de conscientização e educação, sobre diversas temáticas e que chamassem a atenção do homem, conforme o DSC 6 a seguir:

"Eu acho que o caminho é pelas campanhas. Primeiro, a gente deve estudar e ver o que vai fazer, e depois informar e orientar esses homens. Campanhas de conscientização e de educação, isso eu acho que era o fundamental. Implementar um evento que chamasse a atenção do homem, para que ele, interessado por esse evento, chegasse ao serviço de saúde, sensibilizando a população masculina com trabalhos de educação em saúde e ações de promoção da saúde e prevenção de doenças, como palestras sobre alcoolismo, drogas, violência domestica, CA de próstata, doenças crônicas. Acompanhar os homens que já estão doentes. E também falar sobre os direitos deles" (DSC 6, IC 2).

Segundo o Ministério da Saúde ${ }^{(8)}$, os homens podem ser sensibilizados para se tornarem responsáveis pelo seu cuidado e da sua família por meio das ações educativas, proporcionando a diminuição e até mesmo o rompimento da barreira existente entre homens e unidades de saúde.

A IC 3 (Quadro VI) considera a importância de estimular e buscar esses homens, indo onde eles estão e sensibilizando-os através, principalmente, dos agentes comunitários de saúde e líderes comunitários.

Em um estudo(21) com profissionais e usuários de serviços de saúde de três municípios em três regiões brasileiras (Nordeste, Sudeste e Sul), que tinham alguma experiência com atividades envolvendo homens na atenção básica, os profissionais entrevistados enfatizaram a oportunidade de incentivar e potencializar a participação do homem nos cuidados à saúde, para tanto, não podem perder a chance de prevenir doenças, agravos e estimular o homem para o cuidado com a sua saúde.

Pode-se citar algumas estratégias para avanço na prevenção e promoção da saúde dos homens: o deslocamento dos profissionais de saúde para o atendimento dos homens em seus locais de trabalho, atendimento à demanda especifica por contracepção e educação em saúde com a elaboração de cartilhas educativas ${ }^{(21,23)}$.

Já em relação à IC 4 (Quadro VI), apresenta-se a ideia da inclusão da saúde do homem no calendário de atendimento da equipe de saúde da família, com um atendimento específico ao público masculino. Dessa maneira, os serviços têm que se adequar, de certa forma, a essa população, e assim conseguirão atraí-la às unidades de saúde, mas antes serão necessárias algumas mudanças, como a ampliação do horário de funcionamento da $\mathrm{UBS}^{(28)}$.
Na IC 5 (Quadro VI), a importância da capacitação dos profissionais de saúde sobre a PNAISH é apontada para que possa ser trabalhada a saúde do homem de maneira integral, atendendo à necessidade da população masculina. Observase que o apoio da gestão é de primordial importância no processo de superação das dificuldades apontadas neste estudo, uma vez que, por meio dela, tornam-se possíveis os espaços de promoção da saúde masculina e da prevenção de doenças. Por sua vez, o homem deve reconhecer a importância dos cuidados de sua saúde e perceber a UBS como espaço adequado para a consagração de tal objetivo.

O cuidado do homem é processo dinâmico e complexo, tendo a PNAISH fundamental importância para o acolhimento da população masculina nos serviços de saúde. Porém, é importante mencionar que a referida política deve ser adequada às reais necessidades da comunidade, com uma maior divulgação aos profissionais da saúde, principalmente da Estratégia Saúde da Família, tornando a atenção básica verdadeira porta de entrada do sistema de saúde para o homem.

Este estudo possui como limitações a restrição da análise aos profissionais da saúde ter sido realizado em apenas uma UBS, o que dificulta a generalização dos resultados, não obstante, a partir deste artigo, novas abordagens e estratégias podem ser realizadas através dos apontamentos destacados pelos profissionais, assim como uma maior abrangência sobre o conhecimento da PHAISH, pois é a integralidade da saúde do homem um desafio na atenção básica.

\section{CONSIDERAÇÕES FINAIS}

Ficou evidenciado que grande parte dos profissionais entrevistados não conhecem a PNAISH e poucos compreendem a sua importância para o acompanhamento integral do homem. Dessa forma, a deficiente instrução dos profissionais sobre essa política demonstra a possível ausência de capacitações acerca da saúde do homem, o que influencia diretamente a assistência a esse público-alvo.

A partir do presente trabalho, dessume-se que os profissionais da saúde da UBS pesquisada consideram importante a saúde do homem, porém, há pouco incentivo para se trabalhar com esse público, que necessita de atenção e cuidado por parte da equipe de saúde. Ademais, durante muito tempo, o homem ficou às margens das políticas públicas no Brasil.

Percebe-se ainda, através de discursos dos profissionais, que há várias dificuldades para a implementação da PNAISH, como a deficiência de recursos materiais e humanos capacitados para implementação de mais uma política, bem como a falta de interesse do próprio 
homem em cuidar da sua saúde. Dessa forma, são várias as dificuldades apontadas pelos profissionais, as quais necessitam ser analisadas e solucionadas, principalmente o apoio da gestão em saúde e reorganização das ações de saúde, como preconizado pela política.

Durante a elaboração deste estudo, verificou-se que a UBS realiza ações pontuais para o público masculino, na maioria das vezes não direcionadas especificamente ao homem, desconsiderando as reais necessidades desse público-alvo e tampouco os indicadores de saúde referenciados na PNAISH. Destarte, faz-se necessário um programa de educação continuada que capacite e sensibilize os profissionais sobre a PNAISH, pois possibilitará a implementação efetiva da mencionada política.

Não obstante, mesmo diante das dificuldades apresentadas, os profissionais entrevistados destacaram ser possível a implementação da PNAISH, pois uma equipe comprometida e empenhada é capaz de solucionar os problemas, tornando possível para a comunidade o acesso à saúde com qualidade.

\section{REFERÊNCIAS}

1. Silva DRG, Vasconcelos TB, Vidal MPB. Olhe para mim, escute-me: necessidades em saúde de homens. Rev Baiana Saúde Pública. 2013;37(4):835-51.

2. Burille A, Gerhardt TE. Doenças crônicas, problemas crônicos: encontros e desencontros com os serviços de saúde em itinerários terapêuticos de homens rurais. Saúde Soc. 2014;23(2):664-76.

3. Dutra DG, Faria MJG, Carneiro SS, Rodrigues MAR. Saúde do homem: uma visão sociocultural sobre a Política Nacional de Atenção Integral a Saúde do Homem. In: III Seminário de Pesquisas e TCC da Faculdade União de Goyazes; 2012. Trindade: FUG; 2012.

4. Silva PAS, Furtado MS, Guilhon AB, Souza NVDO, David HMSL. A saúde do homem na visão dos enfermeiros de uma unidade básica de saúde. Esc Anna Nery Rev Enferm. 2012; 16(3):561-8.

5. Ramalho MNA, Albuquerque AM, Maia JKF, Pinto MB, Santos NCCB. Dificuldades na implantação da Política Nacional de Atenção Integral à Saúde do Homem. Ciênc Cuid Saúde. 2014;13(4):642-9.

6. Faria MA, Paula DMP, Martins AM, Miranda JJ, Neves-Silva P, Rodrigues T, et al. Política Nacional de Atenção Integral à Saúde do Homem: percepções de gestores de unidades básicas de saúde de Belo Horizonte-MG. Espaç Saúde. 2015;16(3):5-13.
7. Leal AF, Figueiredo WS, Silva GSN. O percurso da Política Nacional de Atenção Integral à Saúde dos Homens (PNAISH), desde a sua formulação até sua implementação nos serviços públicos locais de atenção à saúde. Ciênc Saúde Coletiva. 2012;17(10):2607-16.

8. Ministério da Saúde (BR), Secretaria de Atenção à Saúde, Departamento de Ações Programáticas Estratégicas. Política nacional de atenção integral à saúde do homem: princípios e diretrizes. Brasília: Ministério da Saúde; 2009.

9. Separavich MA, Canesqui AM. Saúde do homem e masculinidades na Política Nacional de Atenção Integral à Saúde do Homem: uma revisão bibliográfica. Saúde Soc. 2013;22(2):415-28.

10. Minayo MCS. O desafio do conhecimento: pesquisa qualitativa em saúde. $8^{\text {a }}$ ed. São Paulo: Editora Hucitec/ Rio de Janeiro: ABRASCO; 2004.

11. Fontanella BJB, Ricas J, Turato ER. Amostragem por saturação em pesquisas qualitativas em saúde: contribuições teóricas. Cad Saúde Pública. 2008;24(1):17-27.

12. Leopardi MT, Beck CLC, Nietsche EA, Gonzales RMB. Metodologia da pesquisa na saúde. Santa Maria: Pallotti; 2001.

13. Lefèvre F, Lefèvre AMC. Discurso do sujeito coletivo: um novo enfoque em pesquisa qualitativa (desdobramentos). 2a ed. Caxias do Sul: Educs; 2005.

14. Cavalcanti YW, Padilha WWN, Paulino MR, Moreira MSC. Motivações, práticas e percepções de cirurgiõesdentistas sobre o trabalho na Atenção Básica de João Pessoa - PB. RFO UPF. 2010;15(3):228-32.

15. Schraiber LB, Gomes R, Couto MT. Homens e saúde na pauta da Saúde Coletiva. Ciênc Saúde Coletiva. 2005;10(1):7-17.

16. Figueiredo WS. Assistência à saúde dos homens: um desafio para os serviços de atenção primária. Ciênc Saúde Coletiva. 2005;10(1):105-9.

17. Carrara S, Russo JA, Faro L. A política de atenção à saúde do homem no Brasil: os paradoxos da medicalização do corpo masculino. Physis (Rio J). 2009;19(3):659-78.

18. Santos SMP, Medeiros TFR, Sousa FLP, Santos LV, Mariz SR, Xavier AG. Professional practices in reproductive planning at the family health strategy. Rev Enferm. UFPE Online. 2015;9(Suppl 7):9046-52.

19. Teixeira DC, Brambilla DK, Adamy EK, Krauzer IM. Concepções de enfermeiros sobre a política nacional de 
atenção integral à saúde do homem. Trab Educ Saúde. 2014;12(3):563-76.

20. Mendonça VS, Andrade NA. A Política Nacional de Saúde do homem: necessidade ou ilusão? Rev Psicol Polít. 2010;10(20):215-26.

21. Moreira MCN, Gomes R, Ribeiro CR. E agora o homem vem?! Estratégias de atenção à saúde dos homens. Cad Saúde Pública. 2016;32(4):e00060015.

22. Gomes R. Sexualidade masculina e saúde do homem: proposta para uma discussão. Ciênc Saúde Coletiva. 2003;8(3):825-9.

23. Martins AM, Malamut BS. Análise do discurso da Política Nacional de Atenção Integral à Saúde do Homem. Saúde Soc. 2013;22(2):429-40.

24. Schwarz E. Reflexões sobre gênero e a Política Nacional de Atenção Integral à Saúde do Homem. Ciênc Saúde Coletiva. 2012;17(10):2579-88.

25. Francischini AC, Moura SDRP, Chinellato, M. A importância do trabalho em equipe no programa saúde da família. Investigação. 2008;8(1-3):25-32.

26. Souza ECF, Vilar RLA, Rocha NSPD, Uchoa AC, Rocha PM. Acesso e acolhimento na atenção básica: uma análise da percepção dos usuários e profissionais de saúde. Cad Saúde Pública. 2008;24(Supl 1):S10010 .
27. Pinafo E, Nunes EFPA, González AD, Garanhani ML. Relações entre concepções e práticas de educação em saúde na visão de uma equipe de saúde da família. Trab Educ Saúde. 2011;9(2):201-21.

28. Knauth DR, Couto MT, Figueiredo WS. A visão dos profissionais sobre a presença e as demandas dos homens nos serviços de saúde: perspectivas para a análise da implantação da Política Nacional de Atenção Integral à Saúde do Homem. Ciênc Saúde Coletiva. 2012;17(10):2617-26.

\section{Endereço primeiro autor:}

Liana Maria Rocha Carneiro

Avenida Antônio Justa, 3161

Bairro: Meireles

CEP 60165-080 - Fortaleza - CE - Brasil

E-mail: lianarcarneiro@yahoo.com.br

\section{Endereço para correspondência:}

Thiago Brasileiro de Vasconcelos Rua: Aveledo, 500. Apto. 201 Torre 2.

Bairro: Messejana.

CEP: 60871-210 - Fortaleza, CE - Brasil

E-mail: thiagobvasconcelos@hotmail.com 